\title{
Electrical and spectroscopic characterization of 7-cell Si-drift detectors
}

\author{
K. Hansen ${ }^{\star}$, C. Reckleben, I. Diehl, E. Welter \\ Deutsches Elektronen-Synchrotron DESY, 22607 Hamburg, Germany
}

\begin{abstract}
Ten detector modules based on monolithic 7-cell Si-drift detectors with integrated junction field effect transistors (JFETs) are currently under production. The modules' hexagonal shape with a wrench size of $16 \mathrm{~mm}$ allows very small distances to the samples and a compact multi-module arrangement. The sensors have active areas of $\sim 50 \mathrm{~mm}^{2}$ and a thickness of $450 \mu \mathrm{m}$. A proper spectroscopy operation of all modules was obtained by 5 common supply voltages and a 6 th voltage which must be individually adopted. Detector capacitances varied from $83 \mathrm{fF}$ to $145 \mathrm{fF}$, where statistical spreading caused by device mismatch amounts to $0.4 \%$. On-chip scattering of the JFET's transconductance and source potential in a source follower configuration are around $1 \%$. Their spreading caused by process variations and device mismatch remain below $8 \%$. Typical spectral resolution and non-linearity is about $300 \mathrm{eV}$ and below $1 \%$ between $4.5 \mathrm{keV}$ and $18 \mathrm{keV}$, respectively. After irradiation with a total dose of $\sim 2$ Mrad the resolution decreases by $\sim 40 \%$. By shielding the cell borders and JFETs from direct irradiation with usage of a $\mathrm{Zr}$ mask, a spectral peak-to-valley ratio of $\sim 1000$ was achieved.
\end{abstract}

Key words: Silicon drift detector, X-ray spectroscopy, junction field effect transistor, electrical performance, spectroscopic performance, radiation tolerance 


\section{Introduction}

Experiments with synchrotron radiation, which detect X-ray fluorescence like absorption spectroscopy, standing waves, or fluorescence tomography demand for energy-resolving detectors. For good detection efficiency, it is necessary that these devices are able to observe simultaneously the radiation emitted into a large angular range. With present and future high-brilliance synchrotron radiation sources it is also important that the detectors can cope with high local count rates. At present, this kind of experiments is done using detectors with single-element sensors or voluminous hybrid sensors having a few small-sized sensitive elements, which thus only register a small fraction of the totally emitted radiation. Sensitive sensors are for example especially designed Si-drift diodes (SDDs) [1]. The SDD operates in the room temperature range, whereas conventional $\mathrm{Si}(\mathrm{Li})$ or Ge detectors requires cooling with liquid nitrogen. Recently, square arrangements of 77 and 19 hexagonally shaped drift diodes have been developed to increase the detection area and integral count rate, and thereby the detection efficiency [2;3]. The basic idea of our module concept is to extend the hexagonal single-cell shape to a hexagonal array of seven cells and also a hexagonal module shape. The 7-cell SDD module is of much smaller size than present commercially available instruments and has already shown its mega-count rate capability at room temperature [4]. Its compactness allows very short distances to the specimen. These mod-

$\bar{\star}$ Tel.: $+49-40-8998-3343$; fax: +49-40-8998-4088.

Email address: karsten.hansen@desy.de (K. Hansen). 
ules have perpendicular sidewalls and can be combined in a flexible way to larger arrangements in accordance with the experimental requirements and with the space available in a specific experiment. For many applications it is also advantageous to reach larger spectral peak-to-background ratios. The identification of origins of events with partial charge collection was already a topic for single-cell [5] and 7-cell SDDs [6]. Applying spatially-resolved spectroscopic measurements to sensors of the new 7-cell SDD family utilized in [4] we identified the regions where the charge collection is incomplete. In [7] we reported already on a mask-based approach to reduce this effect.

The objective of this paper is the presentation of the electrical and spectroscopic performance of a small number of sensor modules taking advantage of the mask approach for the 7-cell SDDs with an active area of up to $50 \mathrm{~mm}^{2}$. In section 2, the setup of the module's sensor part is presented and methods for its electrical and spectroscopic characterization are summarized. Section 3 explains in detail the procedures for the extraction of the most important small-signal parameters and presents the results. In particular, sensor-, JFETand interconnection-related parameters will be determined and analyzed. Finally, the improvements of the spectral behaviour are described.

\section{Setup and experiments}

Fig. 1 shows a photograph of the sensor part of the 7-cell SDD modules. The sensor (PNSensor GmbH, Germany) is placed with its downward oriented entrance window on a lower step $(\sim 300 \mu \mathrm{m}$ deep) of a 3-D structured base frame (Hightec MC, Switzerland). AlN wafers of $635 \mu \mathrm{m}$ thickness and a $\mathrm{Cu}$ based metallization in thin-film technique were chosen as base material and 


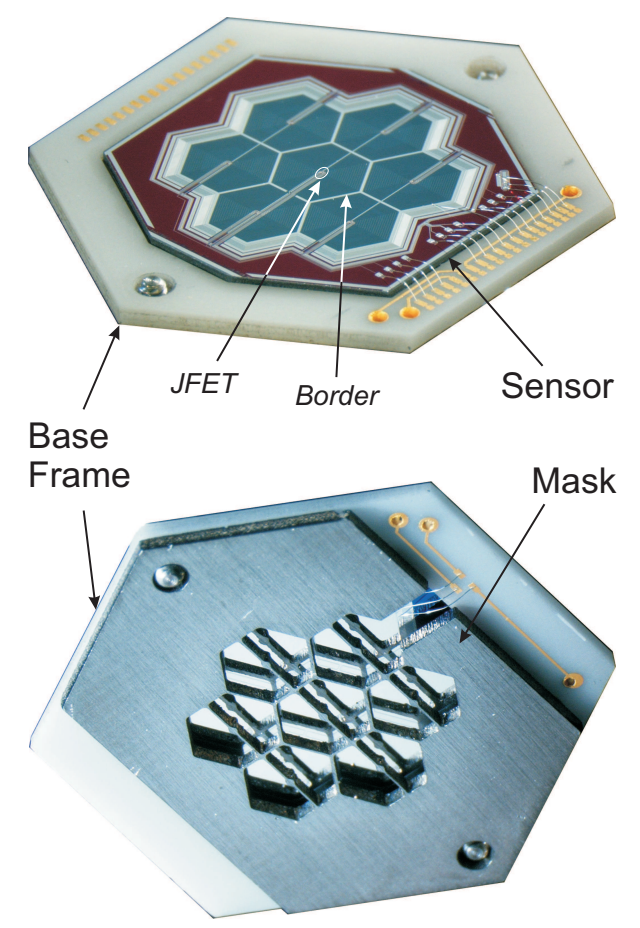

Fig. 1. Photograph of the base frame with 7-cell SDD on top side and mask on back side.

interconnection layers for the double-sided carrier, respectively. Conventional wire-bond technique (AlSi $1 \%, 25-\mu \mathrm{m}$ diameter) was used to connect the output and biasing pads of the sensor to the pad row at the right-hand side of the frame. The sensor needs to be contacted also on the side facing the entrance window (Fig. 1 bottom). Here, the wire loops are located between the side walls of a small channel at the right-hand side of the mask mounted onto the base frame's back side. Through-hole vias are used to bring all lines to the top surface. Zr plates of 450- $\mu \mathrm{m}$ thickness (Goodfellow GmbH, Germany) were chosen as base material of the mask. Zr is ideally suited for photon absorption between $2.5 \mathrm{keV}$ (highest L emission line) and $18 \mathrm{keV}$ (K-edge), which is also the main spectral operation range of the $450-\mu \mathrm{m}$ thick SDD sensor. The straps and bridge-like structures of the mask cover the cell borders and JFETs, 
respectively. The charge collection is incomplete if photons hit either the cell borders or the JFETs which leads always to larger spectral background level at the low-energy side of a spectral line [7]. The mask structure shown in Fig. 1 was produced using laser-cutting technique (Nutech GmbH, Germany). The precise alignment of the mask with respect to the sensor and of the sensor with respect to the base frame was achieved by using a manual flip-chip die bonder (Finetech GmbH, Germany). The dual-component adhesive H70E-175 (Polytec GmbH, Germany) was chosen for gluing procedures. Two holes in the corners of the base frame and mask (cf. Fig. 1) allows a screw connection to further housing parts (not shown).

The SDD principle is based on the idea of sideward depletion, where a $n^{-}$doped substrate is fully depleted by a small sized ohmic $n^{+}$-contact (anode) positively biased with respect to a surrounding planar $p^{+}$-electrode on the substrate's top side and planar $p^{+}$-diode contact on the substrate's back side [1]. By subdividing the $p n$ junction on the top side into 21 concentric hexagonalshaped electrodes and supplying a suitable voltage gradient on such a ring system, a nearly radial symmetrical lateral field is generated, forcing signal electrons radially inwards to the $n^{+}$-ring anode. The $p^{+}$-rings are biased by means of an integrated resistive voltage divider, which reduces the external biasing connections only to the innermost $(\mathrm{R} \# 1)$ and the outermost ring $(\mathrm{R} \# \mathrm{X})$, where the outermost ring is a honeycomb-like structure and common for all cells (cf. Fig. 1 top). In this way, a small anode is defined, whose charge collecting capacitance $C_{\text {anode }}$ is independent of the sensitive cell area of $\sim 7 \mathrm{~mm}^{2}$. The anode is directly connected to the gate $(\mathrm{G})$ of the on-sensor integrated JFET, keeping the total node capacitance $C_{\text {det }}=C_{\text {anode }}+C_{G D}$ as low as $\sim 110 \mathrm{fF}$ (cf. Section 3.2), where $C_{G D}$ is the gate-drain capacitance of the JFET. On 
the back side the $p^{+}$-region is subdivided into two sub regions. The first sub region consists of seven hexagonal-shaped large contacts (cell size) which are connected to each other (contact BC). The second sub region is a honeycomblike structure at the cell boundaries like $\mathrm{R} \# \mathrm{X}$ on the top side. $\mathrm{BC}$ can be slightly positive biased with respect to boundary contact $\mathrm{BC}^{*}$, forcing signal electrons inwards to the main cell area. In this way, split events will also be suppressed "electronically".

The $n$-channel JFETs are separated from the $n^{-}$-bulk by a deep $p$-implantation. This inner guard (IG) contact is negatively biased with respect to the surrounding bulk (inner substrate $n^{+}$-contact: IS) and to the JFET's source ring [8]. Drain D, IG, and IS as well as R\#1 are connected via metal lines to a corresponding bus line surrounding the honeycomb-like outermost ring $\mathrm{R} \# \mathrm{X}$ (cf. Fig. 1 top). The individual source lines are also routed to the peripheral sensor pad row. Additionally to IS, the bulk is also contacted by a honeycomblike ohmic $n^{+}$-contact (outer substrate $n^{+}$-contact: OS) located between R\#X and the bus lines.

The charge-collecting active area of all seven cells is therewith supplied by 6 common voltages, namely $V_{R \# 1}, V_{R \# X}, V_{B C}, V_{B C^{*}}, V_{I S}$, and $V_{O S}$, whereas the JFETs are supplied by the common voltages $V_{D}$ and $V_{I G}$. Adjacent to the peripheral pad row a diode was integrated in order to measure the sensor's temperature (cf. Fig. 1 top: back).

During the project 10 detector modules each one with 7 integrated cells have been fabricated and characterized, where the sensors stem from different wafers and also different process runs. For a proper operation of several modules in parallel it is of practical interest to minimize the number of module-individual 
bias voltages, where the main criterion is the spectral line width. The JFETs have been operated in a source-follower configuration by means of an external current source. Its dynamic range and dc-output level which is determined by $C_{d e t}$ as well as by the JFET's current-voltage characteristics [9] has been evaluated. Furthermore, the pulse response at the output is limited by the load capacitance and therewith also by its parasitic term due to the larger on-sensor trace lengths. All these effects must be considered in the design of the readout electronics. Operating-point parameters like the gate-source voltage $V_{G S}$ and the drain-source voltage $V_{D S}$ as well as small-signal parameters like the JFET's transconductance $g_{m}$ and the drain-source resistance $r_{D S}$ were extracted from current-voltage measurements. The gate-source capacitance $C_{G S}$ and the para-

sitic source-node capacitance $C_{\text {par }}$ were derived from transient measurements, and $C_{\text {det }}$ from spectroscopic measurements. Finally, the linearity, resolution, and peak-to-background behaviour were analyzed at room temperature. The spectroscopic performance was evaluated using a new 7-channel integrated circuit [10] for the sensor readout in front of a data-acquisition (DAQ) system [11] for the analog-to-digital conversion and online-spectral data analysis.

\section{Results}

\subsection{JFET}

The drain current $I_{D}$ of a JFET operating in the triode region can be described by

$$
I_{D}=\beta_{0}\left(1-\alpha_{0} T\right) \cdot\left(V_{G S}-V_{T 0}\right)^{n p} \cdot \tanh \left(\frac{\alpha V_{D S}}{V_{G S}-V_{T 0}}\right) \cdot\left(1-\lambda V_{D S}\right)
$$


where $\beta_{0}$ is the transconductance parameter, $\alpha_{0}$ its linear temperature coefficient, $V_{T 0}$ the pinch-off voltage, $n p$ the power-law exponent, $\alpha$ the triode-tosaturation transition parameter, and $\lambda$ the channel-length modulation parameter of the JFET model level 2 [12]. A separate JFET test structure (cf. left photograph in Fig. 2), from one of the process runs as the 7-cell SDDs stem from, were used to measure the output characteristic of the JFET within a temperature range between $-10^{\circ} \mathrm{C}$ and $30^{\circ} \mathrm{C}$. $V_{G S}$ was varied between $-2 \mathrm{~V}$ and $0 \mathrm{~V}, V_{D S}$ between $0 \mathrm{~V}$ and $12 \mathrm{~V}$. The parameters of (1) were then varied to fit the theoretical output characteristic to the experimental data. $\beta_{0}=$ $111 \mu \mathrm{A} / \mathrm{V}^{1.6}, \alpha_{0}=1.8 \cdot 10^{-3} 1 /{ }^{\circ} \mathrm{C}, V_{T 0}=-2.43 \mathrm{~V}, n p=1.6, \alpha=1$, and $\lambda=5 \cdot 10^{-2} 1 / \mathrm{V}$ was found as optimum parameter set. Between $I_{D}=100 \mu \mathrm{A}$ and $400 \mu \mathrm{A}$ and within a $V_{D S}$-voltage window of $\pm 1 \mathrm{~V}$ around $5 \mathrm{~V}, 6.5 \mathrm{~V}$, and $8 \mathrm{~V}$, the deviations between simulated and experimental curves remain below $8 \%, 3 \%$, and $6 \%$ at $-10^{\circ} \mathrm{C}, 10^{\circ} \mathrm{C}$, and $30^{\circ} \mathrm{C}$, respectively. For drain currents around $240 \mu \mathrm{A}$ an accuracy better than $2 \%$ was obtained. Fig. 2 shows the small-signal parameters $g_{m}$ and $r_{D S}$ derived from the measured output characteristics versus $I_{D}$ for the given three temperatures and drain-source voltages $(\circ, \bullet, \triangle)$. The dashed and solid curves shows a good agreement to measured data, where the most important small-signal parameter $g_{m}$ is almost independent of $V_{D S}$ and $T$ around $200 \mu \mathrm{A}$ and above. Since the gate of the integrated JFET-SDD configuration (cf. right photograph in Fig. 2) is not externally accessible, the drain-to-source channel was controlled by the guard ring contact. As already mentioned, the ring is connected to a deep implantation layer which separates the transistor from the bulk region and can act as an inner gate. We measured the change of $I_{D}$ with increasing $V_{I G}$ at a fixed $V_{D S}$ and determined $g_{m}$ at $240 \mu \mathrm{A}$. In case of $r_{D S}$, we measured $I_{D}$ with increasing drain voltage at the fixed $V_{G S}$ and determined $r_{D S}$ at $240 \mu \mathrm{A}$. 


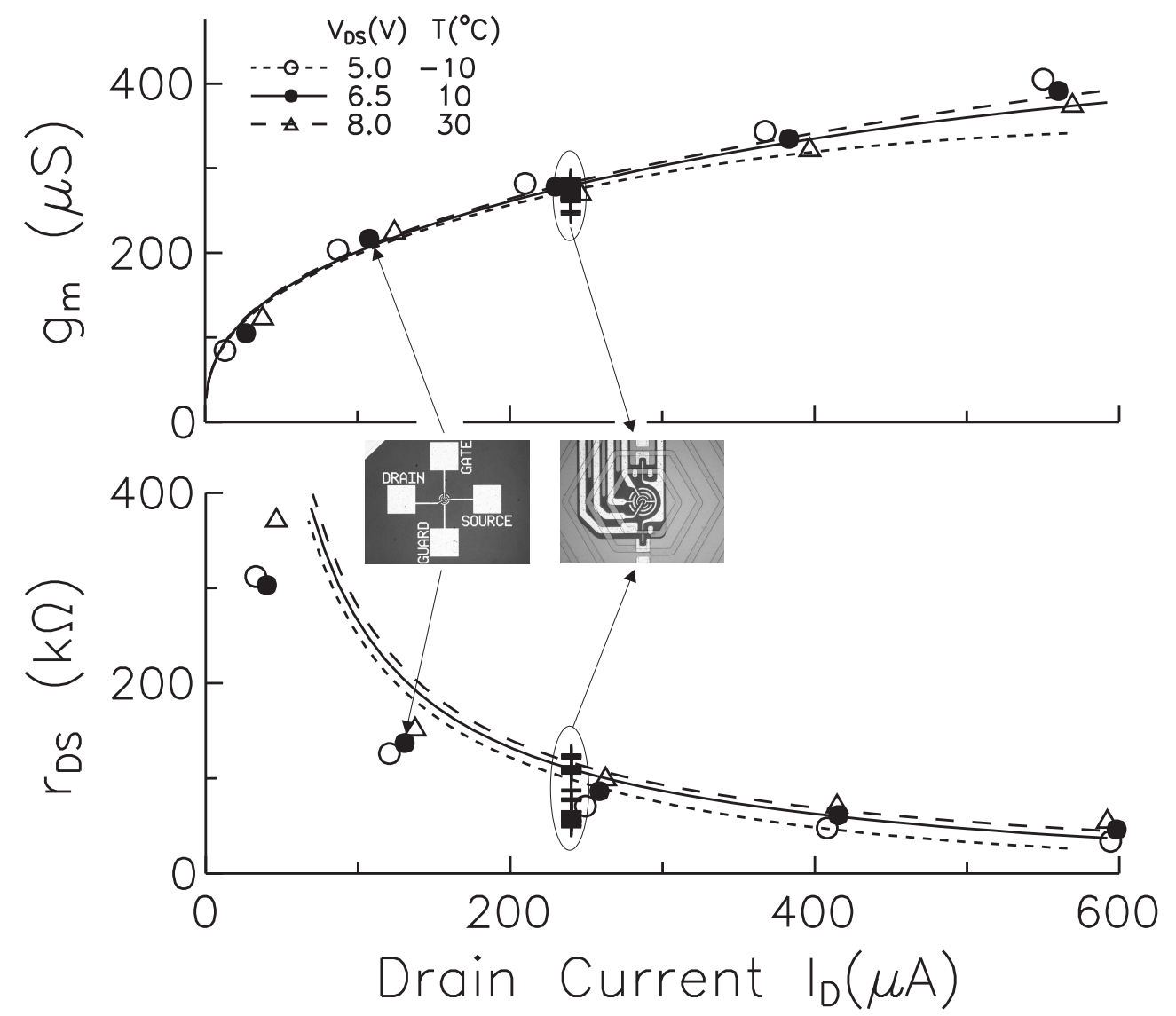

Fig. 2. Transconductance and drain-source resistance of the JFET. Measured values of a JFET test structure and simulated curves are given for $-10^{\circ} \mathrm{C}, 10^{\circ} \mathrm{C}$, and $30^{\circ} \mathrm{C}$. Additionally, measured data of integrated JFETs operated at $I_{D}=240 \mu \mathrm{A}$ and $24^{\circ} \mathrm{C}$ are shown $(+)$.

The values of $g_{m}$ and $r_{D S}$ varied from $245.6 \mu \mathrm{S}$ to $288.2 \mu \mathrm{S}$ and $48.6 \mathrm{k} \Omega$ to $124.8 \mathrm{k} \Omega$, respectively. All 70 measurement points were added in Fig. $2(+)$, they agree closely with the results obtained from the test structure, and can therefore be used for further data analysis. 
The gain of the source-follower configuration is defined by the signal chargeinduced change of source voltage $\Delta V_{S}$ and given by

$$
\frac{\Delta V_{S}}{Q_{d e t}} \simeq \frac{1}{C_{d e t}} \cdot \underbrace{\frac{g_{m} r_{D S}}{\left(1+\frac{C_{G S}}{C_{d e t}}\right)+g_{m} r_{D S}}}_{A_{s f}} \cdot \frac{1}{1+s \cdot \underbrace{\left(1+\frac{C_{G S}}{C_{d e t}}\right) \cdot \frac{C_{C}+C_{p a r}}{g_{m}}}_{\tau_{s f}}},
$$

where $Q_{d e t}$ is the charge collected on $C_{d e t}$, and $A_{s f}$ is the ac-voltage gain for small frequencies of the source follower [13]. The third term on the right-hand side describes its frequency response for frequencies $\omega \ll g_{m} / C_{G S}$ of the complex frequency $s=j \omega \cdot \tau_{s f}$ is the time constant of the load impedance at the source node. The transient behaviour of the source potential was measured using a probe head with a 12-mm needle to contact the source pad. $C_{C}=21.5 \mathrm{pF}$ is the coupling capacitance of the probe-head electronics and considered in parallel to the parasitic capacitance $C_{\text {par }}$ of the source metal line from the JFET in the centre of each cell to the corresponding bond pad (cf. Fig. 1). The transient measurements were performed by irradiating the detector with a ${ }^{55} \mathrm{Fe}$ radioactive source and recording the fluorescence-induced source voltage pulses in order to determine $\tau_{s f}$ for the individual cells. For further details on this method and the procedure to measure the source potentials see [9]. The sum $C_{C}+C_{p a r}$ was then calculated from the measured values of $\tau_{s f}$ and plotted versus the area $A=W \cdot L$ of the metal line, where $W$ and $L$ are its width and length, respectively. Fig. 3 shows an almost linear relationship for two sensors with identical line length but two different widths as stated in the inset. The solid line was fitted to the experimental data by linear regression, where the factor $\left(1+C_{G S} / C_{d e t}\right)$ (cf. $\tau_{s f}$ in $\left.(2)\right)$ was treated as a fitting parameter to minimize the deviations of the experimental data from the linear relationship. During the fit procedure the intersection point 


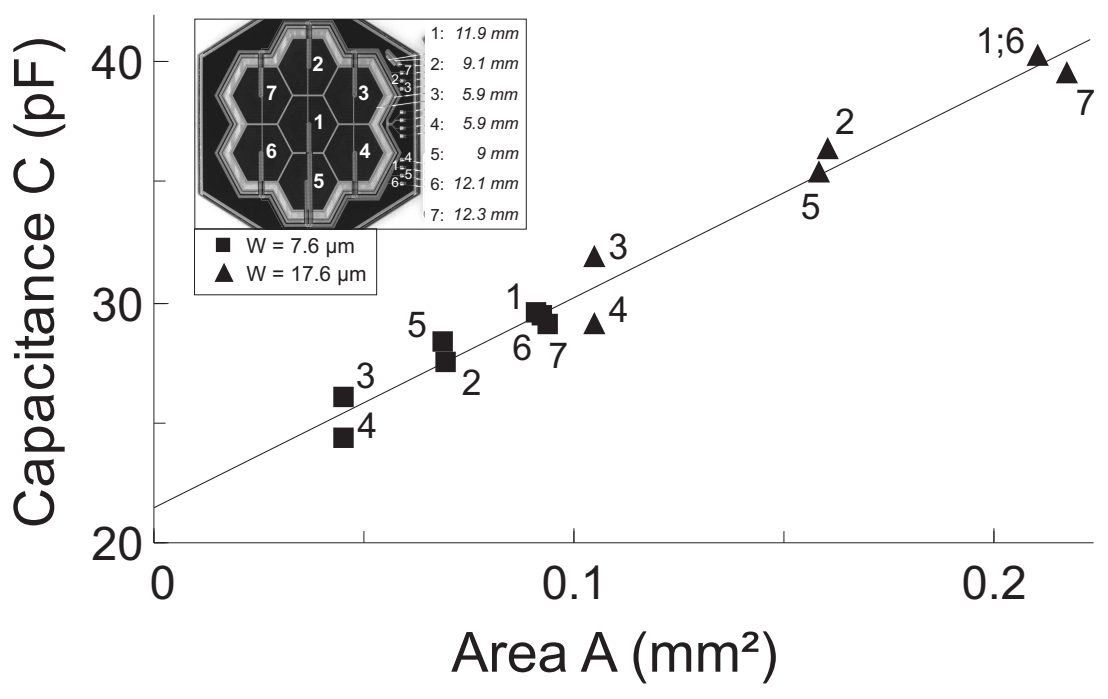

Fig. 3. Source-node capacitance extracted from the measured rise time versus source trace area. Values of a SDD with small (squares) and a SDD with wide (triangles) line widths are shown. The numbers mark the corresponding cells shown in the photograph, and mm-values represent the trace lengths.

of the regression line with the ordinate was fixed at the known value of $C_{C}$. The regression leads to a slope of $87.2 \mathrm{pF} / \mathrm{mm}^{2}$ representing the capacitance per unit area of the source traces. Considering the experimental values of the detector capacitances, the fitting parameter leads to $C_{G S}=67 \mathrm{fF}$.

\subsection{Detector capacitance}

As the mean energy required for the generation of an electron-hole pair in silicon is $w=3.65 \mathrm{eV}[9]$, the charge collected on $C_{\text {det }}$ can be calculated by

$$
Q_{d e t}=-q \cdot \frac{E_{p h}}{w}
$$


where $q$ is the electron charge and $E_{p h}$ the photon energy. Considering the known gain from the readout chip's input to ADC's output, the difference of the peak positions of the $\mathrm{Mn}-\mathrm{K}_{\alpha}$ and $\mathrm{Mn}-\mathrm{K}_{\beta}$ fluorescence lines of the radioactive source were taken to determine $\Delta V_{S}$ at low count rates. Inserting (3) and the above mentioned parameters in (2) allows the final extraction of $C_{d e t}$.

These measurements started always with the determination of an optimal set of bias voltages at room temperature. The JFET's drain was biased at $10 \mathrm{~V}$ and the source was connected to the readout chip-internal current source that draws a constant current of $240 \mu \mathrm{A}$ from the source. A proper operation of all 10 sensors could be reached by the following set of bias voltages: $V_{R \# X}=106.2 \mathrm{~V}, V_{I G}=-16.5 \mathrm{~V}, V_{B C}=-80.6 \mathrm{~V}$, and $V_{B C^{*}}=-83.7 \mathrm{~V} . V_{O S}$

and $V_{I S}$ was set to $0 \mathrm{~V}$ (power ground). Only $V_{R \# 1}$ must be SDD chip-wise adopted between $-19 \mathrm{~V}$ and $-10.5 \mathrm{~V}$ to achieve the maximum spectral resolution. The values of $C_{d e t}$ varied between $83.4 \mathrm{fF}$ and $145 \mathrm{fF}$, where a mean value of $110.9 \mathrm{fF}$ was determined. The on-chip related spread is only $0.4 \%$ (see Table 1).

\subsection{Process variation and device mismatch}

Table 1 summarizes the measured key parameters and voltages obtained for all sensors. Stated are the minimum, maximum, and mean values of 70 data values per parameter. These statistical spreadings are caused by process variations and device mismatch. The last column summarizes the calculated standard deviation of the samples in percent of the mean value. The largest parameter variation belongs to $r_{D S}$, but the influence of $r_{D S}$ on the source-follower's voltage gain is only in the order of a few percent (see (2)). The numbers 
Table 1

Process variation and device mismatch of operating voltages and parameters at $I_{D}=240 \mu A$.

\begin{tabular}{lcccc}
\hline & Min & Max & Mean & Std. Dev. \\
\hline$g_{m}(\mu S)$ & 245.6 & 288.2 & 274 & $3.3 \%(1.4 \%)$ \\
$r_{D S}(k \Omega)$ & 48.6 & 124.8 & 78.6 & $32 \%(1.8 \%)$ \\
$V_{D S}(\mathrm{~V})$ & 7.8 & 10.1 & 9 & $7.8 \%(0.7 \%)$ \\
$V_{G S}(\mathrm{~V})$ & -1.4 & -1 & -1.1 & $9.1 \%(1.8 \%)$ \\
$C_{\text {det }}(\mathrm{fF})$ & 83.4 & 145 & 110.9 & $15.2 \%(0.4 \%)$ \\
$V_{R \# 1}(\mathrm{~V})$ & -19 & -10.5 & -16.1 & - \\
\hline
\end{tabular}

(): device mismatch

in brackets represent the standard deviations only caused by mismatch (on sensor-chip scattering). Compared to the former deviations the on-sensor chip fluctuations are much lower so that it is not necessary to consider a channelwise adoption of the gain.

\section{Spectral performance}

These experiments were carried out with synchrotron radiation at DESY / HASYLAB beam lines C and A1. Using a double-crystal monochromator a calibration standard (NIST 612) and an Au foil were irradiated with monochromatic radiation at $18 \mathrm{keV}$ and $12 \mathrm{keV}$, respectively. In both cases, the detector surface was placed in parallel to the beam axis in order to detect the fluorescence photons only. Additionally, the spatial characterization of the spectroscopic properties of the SDD was done. A fixed photon energy of $9 \mathrm{keV}$ was chosen and the size of the direct beam at the sensor was limited to a di- 
ameter of $4 \mu \mathrm{m}$ for the 1-D and 2-D scans. In all experiments the detector was mounted on a $\mathrm{x}-\mathrm{y}-\mathrm{z}$ translation stage for adjusting purposes and distance variations.

\subsection{Peak-to-background ratio}

The main interest of the spatial characterization was the identification of regions on a detector cell which negatively affect the spectral performance, mainly the peak-to-background ratio [7]. The left graph in Fig. 4 shows the simulated influence of the mask on the peak-to-background ratio by covering the cell borders (96 $\mu \mathrm{m}$ width) and the JFET (280 $\mu$ m diameter). The spectra were calculated from a scan from border to border of the centre cell (cf. inset of Fig. 3, cell 1) through the JFET area. Three cases are shown, a spectrum for total area illumination (top), a spectrum for illumination excluding the border regions (middle), and a spectrum for full coverage of the distorted regions (bottom). Note, that the background levels of the last-mentioned two cases would only be achievable for zero-divergent radiation. From the plots it can be seen that an "ideal" mask can improve the peak-to-background ratio by one order of magnitude. The plot on the right hand side shows fluorescence spectra emitted by the Au sample for different distances to the masked sensor shown in Fig. 1. With increasing distance the background level decreases by a factor of $\sim 5$, and the separation of lines clearly improves. In this case, the mean width of the straps which cover the cell boundaries is $143 \mu \mathrm{m}$, and the mean diameter of the JFET coverage is $290 \mu \mathrm{m}$. The fabrication technique of the Zr-metal mask leads to maximum width tolerances of $\pm 60 \mu \mathrm{m}(16-\mu \mathrm{m}$ standard deviation). Therefore, the mask does not cover all distorted regions 


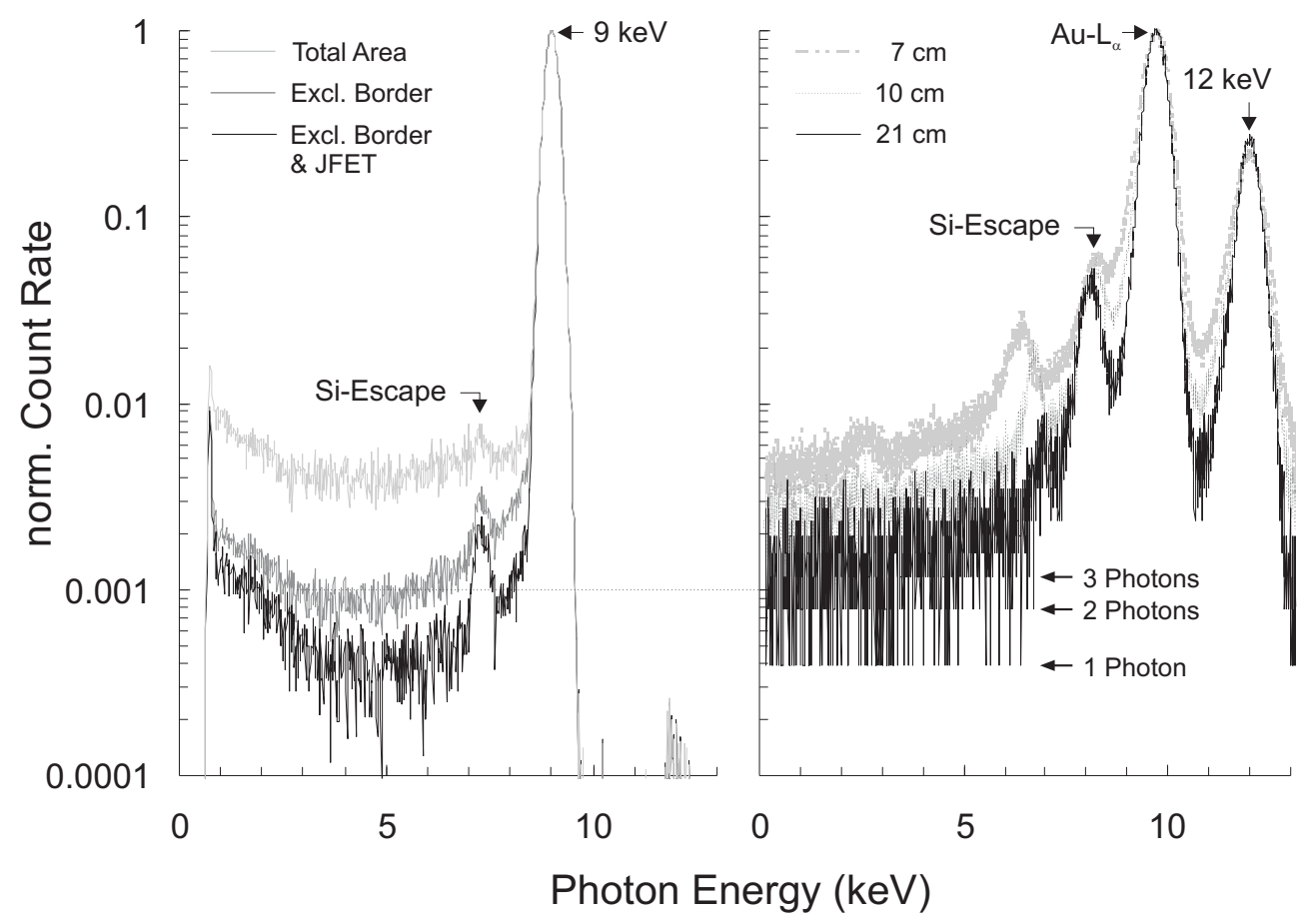

Fig. 4. Simulated normalized spectra (left) for total area illumination (highest background), illumination excluding the borders (medium background), and illumination excluding borders and JFET (lowest background) compared to normalized Au fluorescence spectra (right) of a 7-cell SDD with mask covering all three sensitive areas.

completely. Additionally, we determined a mask displacement of $24 \mu \mathrm{m}$ in $\mathrm{x}$-direction and $55 \mu \mathrm{m}$ in y-direction (direction of bridges holding the JFET coverage) for this module, where a mean mask-to-sensor distance of $403 \mu \mathrm{m}$ was measured. Both types of tolerances give reasons for the reduced peak-tovalley ratio of $\sim 1000$ in "real" experiments, compared with the "ideal" value of $\sim 2000$. 


\subsection{Radiation tolerance}

The radiation tolerance of the biased SDD is verified at room temperature by irradiating detector cell 1 with the direct beam at 17-keV photon energy and a flux of $\sim 3 \cdot 10^{9}$ photons per $\left(\mathrm{s} \mathrm{mm}^{2}\right)$. After each irradiation the beam shutter was closed and a Mn-spectrum was taken by using the ${ }^{55} \mathrm{Fe}$ source. The full-width-half-maximum (FWHM) of the $\mathrm{Mn}^{-\mathrm{K}_{\alpha}}$ line was determined and is displayed in Fig. 5 versus the number of photons. The line width increases linearly from $240 \mathrm{eV}$ up to $320 \mathrm{eV}$ when the detector was irradiated with $10^{14}$ photons. Considering a quantum efficiency of $53 \%, 10^{14}$ photons correspond to an operation period of 2.8 years at a count rate of $600 \mathrm{kcts} / \mathrm{s}$. The total deposited dose was $\sim 2$ Mrad.

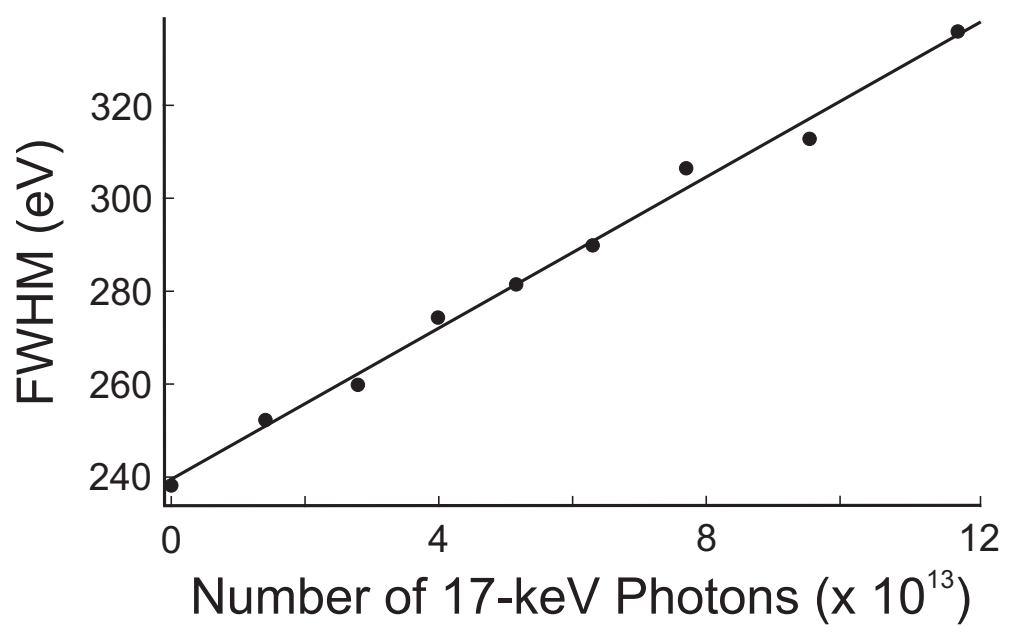

Fig. 5. FWHM after irradiating the SDD versus the number of photons.

\subsection{Linearity and resolution}

Fig. 6 shows the spectra of the calibration standard NIST 612, measured at $24^{\circ} \mathrm{C}$. Cell no. 3 of this detector was not working properly and is rejected in 

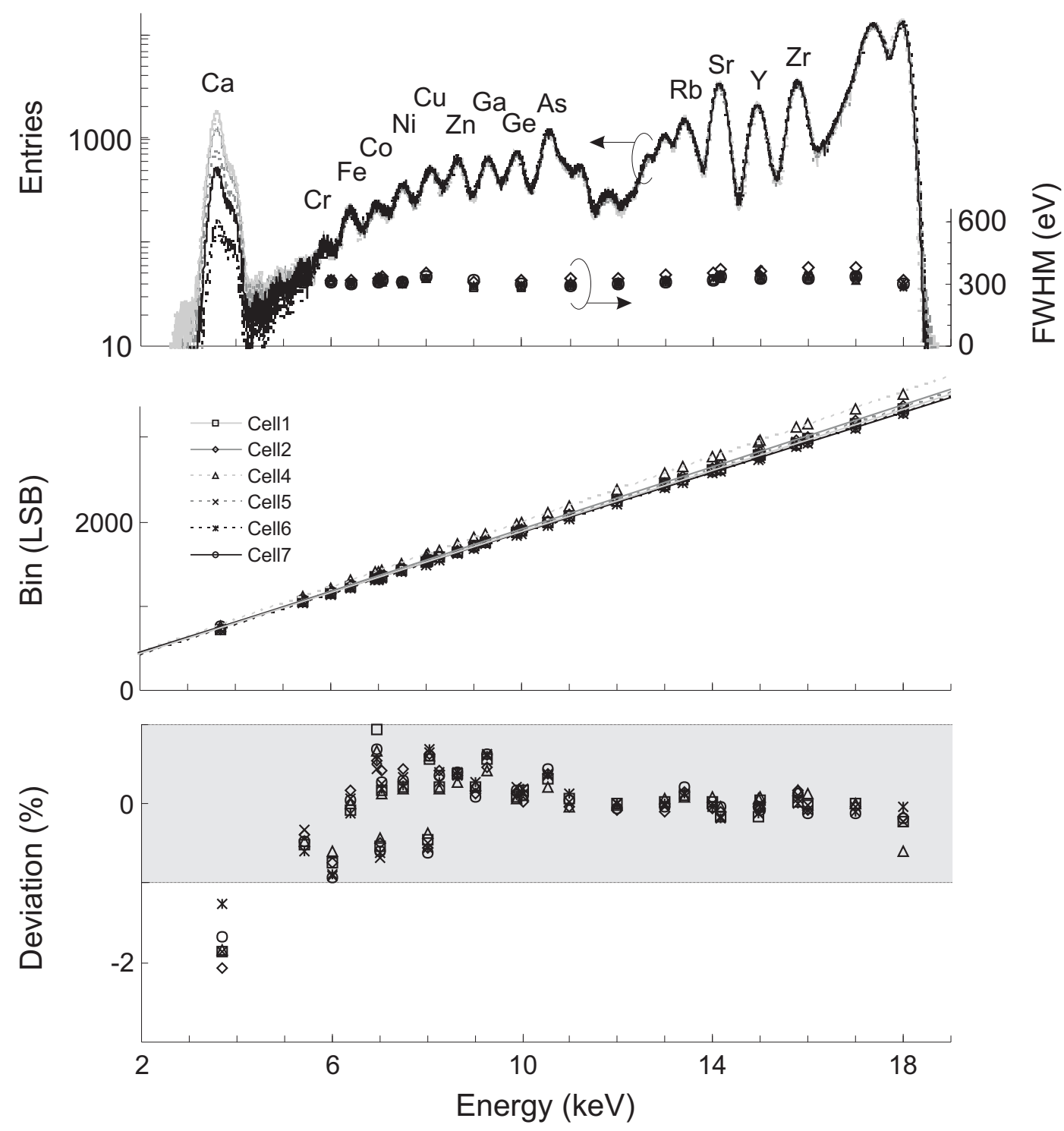

Fig. 6. Fluorescence spectra of the calibration standard NIST 612 (top), linearity characteristics with linear regression lines (middle), and deviation of the measured values from the regression lines (bottom).

Fig. 6. Due to inhomogeneous impurity concentrations in the detector bulk material it was sometimes impossible to find a common supply-voltage set for which all 7 cells could work satisfactorily in parallel [2]. Bad cells typically show a spectrum with a distinct tail on the low energy side of a line with a strong deterioration of its spectral resolution. This problem should be overcome in prospective detector production runs. The remaining 6 cells however 
showed excellent spectra (Fig. 6 top) with a FWHM of $\sim 300 \mathrm{eV}$ at room temperature. Note, that the electronic noise contribution is $\sim 100 \mathrm{eV}$. Fig. 6 (middle) depicts the measured peak positions (symbols) in ADC bins (LSB) versus photon energy and a linear regression line for each detector cell. In addition to the peaks in the spectra of the calibration standard some elastic stray peak-values from excitation energies in separate measurements are considered. The deviation from the linear regression is well within $\pm 1 \%$ between $4.5 \mathrm{keV}$ and $18 \mathrm{keV}$ for all 6 cells.

Fig. 7 shows the measured FWHM line width of the $\mathrm{Cu}-\mathrm{K}_{\alpha}$ emission lines versus the sum count rate of all 6 properly working cells for $24^{\circ} \mathrm{C}$. These data

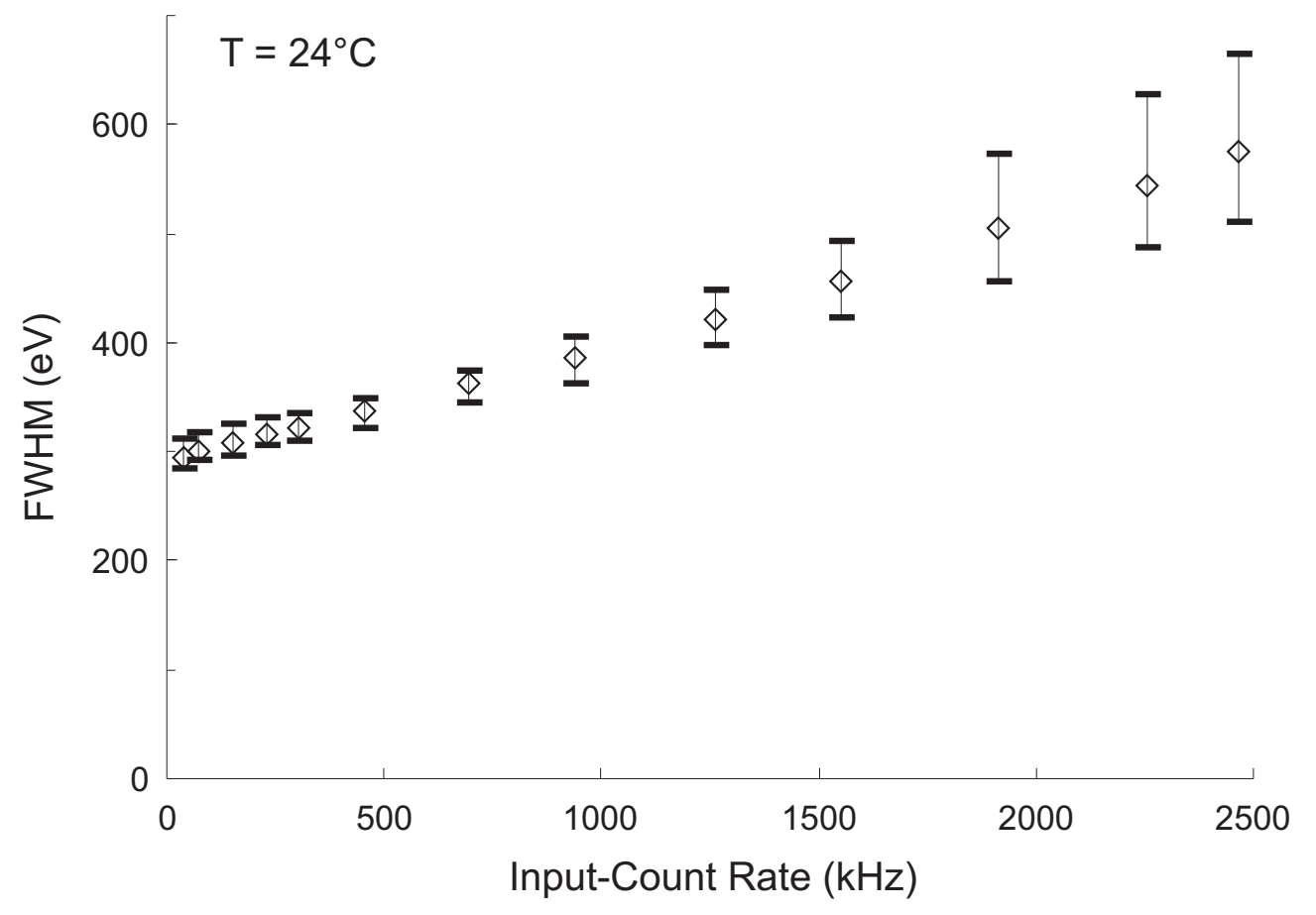

Fig. 7. Measured $\mathrm{Cu}-\mathrm{K}_{\alpha}$ line width versus sum count rate.

suggest that the sensors can be operated at room temperature with megacount rate capability. The bars indicate the minimal and maximal line width of the 6 cells. The achieved resolution is satisfactory for fast counting applica- 
tions with energy discrimination like X-ray absorption spectroscopy or X-ray standing waves.

\section{Summary}

We have realized a small series of 7-cell SDD modules. The experimental characterizations show that the modules meet all requirements in terms of high spectral resolution, high linearity and sufficient radiation tolerance. The peak-to-background ratio has been improved by means of a Zr-mask which covers the cell borders and the JFETs. The compactness of the module and its spectral properties even at room temperature in conjunction with the high count-rate capability makes it a well suited tool for applications in X-ray spectroscopy.

\section{Acknowledgment}

The authors would like to thank H. Klär, E. Wüstenhagen and A. Titze for their help during the measurements as well as A. Venzmer and D. Hammer for module setup, assembly, and tests.

\section{References}

[1] P. Lechner et al., Nucl. Instr. Meth. A458 (2001) 281.

[2] C. Fiorini et al., IEEE Trans. Nucl. Sci. 52 (2005) 1165.

[3] T. Conka-Nurdan et al., IEEE Trans. Nucl. Sci. 51 (2004) 2526. 
[4] C. Reckleben, K. Hansen, I. Diehl, H. Klär, and E. Welter, IEEE NSS 2007, 28th Oct. - 3rd Nov., Honolulu Hawaii, USA, No. 1420

[5] T. Eggert et al., Nucl. Instr. Meth. A512 (2003) 257.

[6] P. Kappen et al., Nucl. Instr. Meth. A467 (2001) 1163.

[7] E. Welter and K. Hansen, 13th Int. Conf. XAFS (XAFS13), Stanford CA, USA (2006) in Proc. AIP XAFS13, CP882 (2007) 917.

[8] C. Fiorini and P. Lechner, IEEE Trans. Nucl. Sci. 46 (1999) 761.

[9] K. Hansen and C. Reckleben, IEEE Trans. Nucl. Sci. 50 (2003) 1718.

[10] I. Diehl, K. Hansen, and C. Reckleben, ESSCIRC 2007, 11th - 13th Sept, Munich, Germany, E2L-G 1133

[11] K. Hansen et al., IEEE Trans. Nucl. Sci. 49 (2002) 541.

[12] Spectre Reference Manual, Rel. 4.3, Cadence Design Systems, Inc., USA, 1994.

[13] M. Sampietro, L. Fasoli, and G. Bertuccio, IEEE Trans. Nucl. Sci. 43 (1996) 2413.

[14] K. Hansen and C. Reckleben, IEEE Trans. Nucl. Sci. 51 (2004) 1283. 